AIDS, Publish Ahead of Print

DOI: 10.1097/QAD.0000000000002660

\title{
The predictors of pain extent in people living with HIV
}

Caroline A SABIN ${ }^{1}$, Richard HARDING ${ }^{2}$, Emmanouil BAGKERIS ${ }^{1}$, Adam GERESSU $^{1}$, Kennedy NKHOMA ${ }^{2}$, Frank A POST ${ }^{3}$, Memory SACHIKONYE ${ }^{4}$, Marta BOFFITO $^{5}$, Jane ANDERSON ${ }^{6}$, Patrick WG MALLON ${ }^{7}$, Ian WILLIAMS ${ }^{1}$, Jaime VERA $^{8,9}$, Margaret A JOHNSON ${ }^{10}$, Daphne BABALIS ${ }^{11}$, Alan WINSTON ${ }^{12}$.

1. Institute for Global Health, UCL, London, UK

2. Florence Nightingale Faculty, Cicely Saunders Institute, Kings College London, UK

3. Caldecot Centre, King's College Hospital, London, UK

4. UK Community Advisory Board (UK-CAB), London, UK

5. St. Stephen's Centre, Chelsea and Westminster Hospital, London, UK

6. Homerton University Hospital, London, UK

7. HIV Molecular Research Group, School of Medicine, University College Dublin, Ireland

8. Elton John Centre, Brighton and Sussex University Hospital, Brighton, UK

9. Brighton and Sussex Medical School, Brighton, UK

10. Ian Charleson Day Centre, Royal Free NHS Trust, London, UK

11. Imperial Clinical Trials unit, Imperial College London, UK

12. Department of Infectious Disease, Imperial College London, London, UK

\section{Address for correspondence:}

Professor Caroline A Sabin

Centre for Clinical Research, Epidemiology, Modelling and Evaluation

Institute for Global Health

UCL, Royal Free Campus

Rowland Hill Street

London NW3 2PF

Tel: 02077940500 ext. 34752

E-mail: c.sabin@ucl.ac.uk 
Funding support: This work was supported by investigator-initiated grants from BMS, Gilead Sciences, Janssen, MSD and ViiV Healthcare. Analysis of the pain data received additional financial support from the National Institute for Health Research through a grant application to Professor R. Harding (RP-DG-0517-10004). We acknowledge the use of the NIHR/Wellcome Trust Clinical Research Facility at King's College Hospital. The research is also supported by the National Institute for Health Research (NIHR) Biomedical Research Centre based at Imperial College Healthcare NHS Trust and Imperial College London and by an NIHR Senior Investigator Award to Professor C. A. Sabin.

\section{Abstract}

Objective: To investigate the prevalence of widespread pain among people with HIV (PWH) and describe associations with antiretroviral therapy (ART) and markers of HIV disease stage.

Design: Cross-sectional analysis of cohort study in the UK and Ireland.

Methods: Pain information was collected during the baseline visit (conducted from 2013-2015) through a self-completed mannikin identifying pain at fifteen sites from five body regions. Pain was classified as widespread if reported at $\geq 4$ regions and $\geq 7$ sites, or regional otherwise. Chi-squared tests, Kruskal-Wallace tests and ordinal logistic regression were used to consider associations between pain extent and sociodemographic and HIV-related factors.

Results: Among the 1207 participants $(614$ PWH $\geq 50$ years, 330 PWH<50 years, 263 HIV-negative controls $\geq 50$ years), pain was most commonly reported at the upper (left: 28.9\%, right: $28.0 \%$ ) and lower (left: 25.7\%; right: $24.5 \%$ ) leg, upper (18.6\%) and lower $(29.7 \%)$ back and shoulders (left: $16.0 \%$; right: $16.8 \%$ ). Widespread pain was more commonly reported in PWH than in HIV-negative controls (PLWH $\geq 50$ years: 18.7\%; PLWH $<50$ years: $12.7 \%$; HIV-negative $\geq 50$ years: 9.5\%) with regional pain reported in $47.6 \%, 44.8 \%$ and $49.8 \%$, respectively (global $\mathrm{p}=0.001$ ). In multivariable analyses, pain extent was greater in those with lower educational attainment, those 
exposed to more ART drugs, and those with a higher current CD4 count but longer exposure to immunosuppression.

Conclusions: Widespread pain is commonly reported in PWH and is associated with longer duration of exposure to HIV, immunosuppression and ART. Our findings call for greater awareness, and interventions to support the management, of pain in $\mathrm{PWH}$.

Key words: widespread pain; HIV infection; immunosuppression; risk factors; antiretroviral therapy

\section{Introduction}

The widespread use of antiretroviral treatment (ART) means that people with HIV (PWH) in many parts of the world now have a near-normal life expectancy [1,2]. However, the cohort of PWH is ageing which is resulting in an increase in the prevalence of age-associated co-morbidities [3]. Moderate or severe pain is commonly reported among PWH and is associated with poorer quality-of-life, ART outcomes and engagement in HIV care [4, 5]. Despite this, little research has been undertaken on chronic pain in the modern ART era among individuals in whom HIV infection is largely controlled.

In a recent analysis of the Pharmacokinetic and clinical Observations in PeoPle over fiftY (POPPY) study [6], PWH over the age of 50 years experienced higher rates of pain than similarly aged HIV-negative participants, even after adjustment for confounders. Pain was associated with lower employment and with high levels of healthcare resource use, higher depressive symptoms, poorer quality-of-life and poorer functional status, regardless of age.

There are well documented associations between pain and some of the earlier ART drugs, particularly those from the nucleoside reverse transcriptase inhibitor (NRTI) class, driven by metabolic toxicities [7, 8]. However, findings to support an association of pain with more advanced HIV infection (as expressed by lower current/nadir CD4 counts, higher HIV viral loads and/or longer duration of HIV infection) are inconsistent [9-15]. 
Previous POPPY analyses concentrated only on the presence or absence of pain, and primarily on a comparison between PWH and HIV-negative controls rather than on an investigation of HIV-related associated factors. Here we describe the body distribution of pain in study participants focussing particularly on the prevalence of widespread pain and its associations with socio-demographic factors and markers of HIV disease stage among PWH in the POPPY Study.

\section{Methods}

POPPY is a prospective cohort study at seven clinical sites in the UK and one in Ireland that aims to investigate the impact of HIV on the development and outcomes of comorbidities and pharmacotherapy among older PWH [16]. Three sub-groups are studied: older HIV-positive (age $\geq 50$ years, $n=699$ ), younger HIV-positive (age <50 years, $n=374$ ) and older HIV-negative (age $\geq 50$ years, $n=304$ ) people. Eligible HIVpositive participants acquired HIV through sexual transmission (sex between men or sex between men and women; those acquiring HIV through other routes, including injection drug use, were excluded), were cisgender, and were either of white or black African ethnicity. Those recruited to the younger group of PWH were frequency-matched to the group of older PWH on gender, ethnicity, sexual orientation and participating clinic. HIV-negative participants were required to have a documented negative HIV test; this group was frequency-matched to the older PWH group on age, gender, ethnicity, sexual orientation and geographical location. The study was approved by the UK National Research Ethics Service (NRES; Fulham, London, UK; reference number 12/LO/1409) and written informed consent was obtained from all participants. The present analyses used cross sectional data from the baseline POPPY visit conducted between April 2013 and February 2016.

The POPPY dataset includes information on socio-demographics, pharmacotherapy, personal and family medical history, healthcare utilisation and quality-of-life. The POPPY dataset is linked to the UK Collaborative HIV Cohort (UK sites [17]) and to the UCD ID Cohort (Dublin [18]) for historic data on ART, CD4 counts and HIV RNA. Pain information was collected through a self-completed pain mannikin identifying affected body sites [as in 14]. Pain was classified as widespread based on the 2019 
American College of Rheumatology/Fibromyalgia criteria [19], in which widespread pain was defined if the presence of pain was reported in at least four of the five body regions [(1) neck/upper back/lower back; (2) left and (3) right shoulder/upper arm/lower arm; (4) left and (5) right hip/upper leg/lower leg] and also in at least 7 of 15 distinct body sites (see Table 1). Where pain was present but did not meet these criteria, it was classified as regional.

\section{Statistical analysis}

Comparison of the prevalence of pain at each body site, the extent of pain (widespread, regional, absent) and the number of sites/regions affected between the three POPPY sub-groups was undertaking using Chi-squared and Kruskal-Wallis tests. The same approach was used to consider associations between pain extent and socio-demographic and HIV-related factors in the subgroup of PWH. Socio-demographic factors considered were: age, gender, mode of HIV acquisition, race, body mass index (BMI), educational attainment (low ('O' levels or lower), medium ('A' levels or equivalent) and higher (higher/further education)) and calendar year. ART-related factors considered were the total number of ART drugs received and the years since ART was started. Non-ART HIV-related factors were the latest and nadir CD4 counts (each categorised as $<200, \geq 200-<350, \geq 350-<500$ and $\geq 500$ cells $/ \mathrm{mm}^{3}$ ), the years of exposure to immunosuppression (number of years spent with a CD4 count $<200$ cells $/ \mathrm{mm}^{3}$ ) and the maximum HIV RNA attained (categorised as $\leq 3,>3-\leq 4,>4-\leq 5$ and $>5 \log _{10}$ copies $/ \mathrm{ml}$ ).

Factors that were significantly associated with the extent of pain in univariate analyses were then considered for inclusion included in an ordinal logistic regression analysis to identify independent predictors of widespread and regional pain (quantitative covariates were retained in their continuous form for these analyses). Our aim was to develop a parsimonious model and thus a stepwise selection process was used with factors being removed from the model if they failed to demonstrate a significant association with pain $(\mathrm{p}<0.10)$ at any stage. All analyses were performed using SAS v9.3 (SAS Institute Inc., Cary, NC). 


\section{Results}

Pain mannikins were completed by 1261/1377 POPPY participants (91.6\%; 92.3\% of older PWH, 90.9\% of younger PWH and 90.8\% of HIV-negative controls). After excluding those with missing or inconsistent data $(n=54)$, the final dataset included 1207 participants (Table 1). Those included were more likely to be white MSM and had a known employment status than those not included, with no large differences in age, BMI or the year of visit.

\section{Bodily sites and prevalence of widespread pain}

The most commonly affected sites were the upper leg (left: $28.9 \%$; right: $28.0 \%$ ), lower leg (left: $25.7 \%$; right: $24.5 \%$ ), upper (18.6\%) and lower (29.7\%) back and the shoulders (left: 16.0\%; right: 16.8\%) (Table 2). Pain was more commonly reported in PWH at most sites and regions than in HIV-negative controls. The median (interquartile range, IQR) number of sites affected was $2(0-5)$ in older PWH, $1(0-4)$ in younger PWH and 1 (0-3) in HIV-negative controls ( $\mathrm{p}=0.002$, Kruskal-Wallis test). Widespread pain was reported by 115 (18.7\%), $42(12.7 \%)$ and 25 (9.5\%) of those in the three groups, respectively, with regional pain being reported by 292 (47.6\%), 148 (44.8\%) and 131 (49.8\%) (global $\mathrm{p}=0.001$, Chi-squared test).

Associations with extent of pain and socio-demographic and HIV-related factors in $P W H$

Of the 944 PWH, 907 had longitudinal data on ART exposure and were included in subsequent analyses (Table 1). Univariate analyses revealed that widespread pain was more common in those of white ethnicity $(\mathrm{p}=0.009), \operatorname{MSM}(\mathrm{p}=0.02)$, those with lower educational attainment $(\mathrm{p}=0.009)$ and older individuals $(\mathrm{p}=0.004)$ (Table 3$)$.

Participants had been exposed to a median (IQR) of 6 (4-9) ART drugs and had received ART cumulatively for 9.9 (5.2-16.5) years. The median (IQR) CD4 and nadir CD4 count at study visit were $637(475-830)$ and $188(87-280)$ cells $/ \mathrm{mm}^{3}$ respectively. Just over half $(448,51.4 \%)$ of participants had never had a CD4 count $<200$ cells $/ \mathrm{mm}^{3}$; of the remaining participants, 297 (34.1\% of total) had immunosuppression for $\leq 2$ years, $56(6.4 \%)$ for $>2$ to $\leq 4$ years and $70(8.0 \%)$ for $>4$ years. The median (IQR) maximum 
viral load attained over follow-up was $5.1(4.3,5.6) \log _{10}$ copies/mL, with $829(91.4 \%)$ currently having a suppressed ( $\leq 50$ copies $/ \mathrm{mL})$ viral load.

Those with widespread pain had been exposed to a greater total number of ART drugs $(p=0.0001)$ and had been receiving ART for a longer period of time $(p=0.0001$, Figure 1). Univariate analyses suggested that whilst there was no strong association between the extent of pain and either the latest or nadir CD4 count, those who had experienced prolonged immunosuppression were more likely to be experiencing widespread pain $(\mathrm{p}=0.0002)$. There did not appear to be a strong association with the maximum viral load measured.

In the final multivariable model, educational attainment was the only sociodemographic factor which remained significantly associated with pain extent, with those with the lowest educational attainment having an odds of regional or widespread pain that was 50\% higher than those with high educational attainment. Of the HIV factors, those who had received a greater number of ART drugs in total were more likely to experience regional or widespread pain with the odds of either increasing by $12 \%$ per additional drug. In addition, after adjustment for these previous factors, both a higher current CD4 count and a longer duration of immunosuppression (Table 4) were associated with an increased odds of widespread pain.

\section{Discussion}

In this large cohort of PWH, widespread pain was reported in $18.7 \%$ of $\mathrm{PWH}$ aged $\geq 50$ years and $12.7 \%$ of PWH at younger ages, substantially higher rates than those seen in demographically and lifestyle similar HIV-negative controls (9.5\%). In contrast, rates of regional pain were similar in all groups. Factors associated with pain extent were largely related to HIV infection, with associations with both ART exposure and immunosuppression. Pain among PWH is associated with depressive symptoms, quality-of-life and functional impairment, regardless of age, and has the potential to have a negative impact on HIV-related outcomes [4, 6, 12]. Thus, our study findings have implications for current optimal treatment and care of PWH.

A high prevalence of widespread pain in PWH has been reported in several studies. In one study of 238 PWH in New England [12], pain in multiple sites or all over the body 
was reported by $35 \%$ and $47 \%$ of participants with mild or moderate/severe chronic pain, respectively, compared to only $18 \%$ of those with acute pain. Results from a systematic review of 28 published papers [20] suggested that pain in PWH is most commonly reported in the lower limbs, with $40 \%$ of participants having at least 1 and $17 \%$ having multiple chronic pain diagnoses. Jiao [21] reported that musculoskeletal pain (joint pain and muscular pain, including fibromyalgia) was the most common type of chronic pain, affecting $38 \%$ of all study patients, followed by neuropathic pain $(11 \%)$, headaches $(6 \%)$ and chronic pelvic pain (1.6\%). Notably, $10 \%$ of participants reported other poorly defined pain syndromes.

Although the prevalence of widespread pain has been reported previously, our study advances the field through its comparison with a lifestyle and demographically-similar group of HIV-negative participants, and through its detailed assessment of longitudinal information on immunosuppression and ART exposure. Our findings suggest that longer exposure to a low CD4 count is a risk factor for widespread pain. Published associations between pain and the CD4 count are inconsistent. In a sub-study of the Research on Access to Care in the Homeless (REACH) study, pain, whilst highly prevalent (91.2\%), was not associated with either the CD4 count or CD4 nadir [22]. Similarly, in PWH with alcohol-use disorders recruited to the HIV-LIVE study [23], no association was reported between pain interference and a CD4 count $<200$ cells $/ \mathrm{mm}^{3}$. In contrast, women with HIV in the Women's Interagency HIV Study [15] with a CD4 count $<200$ cells $/ \mathrm{mm}^{3}$ were more than twice as likely to report more severe pain, and more than 50\% more likely to report more frequent pain, than those with higher CD4 counts. Among 317 PWH in San Francisco, pain was associated with the CD4 count [24]. Interestingly, Martin [25] reported a strong association between the number of concurrent pain sites and the CD4 count in PWH who did not report injection drug use (IDU), but no such correlation in IDU. This inconsistency was noted by Parker [20] who reported a higher prevalence of pain in participants with advanced disease in only some of the studies in their systematic review. Of note, our associations with the current and nadir CD4 counts only became apparent after adjustment for ART exposure. Thus, part of the inconsistency in published reports may reflect the fact that associations with immune status may differ between those newly starting ART and those who have been previously exposed to ART for some time and may also differ depending on the 
definition of pain used, as reported in other studies [26,27]. We did not see any association with viral load in the POPPY cohort, although the majority (91.4\%) of individuals in the study were virally suppressed at the study visit.

Our findings suggesting that widespread pain is associated with greater exposure to ART should be interpreted with some caution as our measures of cumulative exposure to ART and the total number of prescribed ART drugs may act as proxy markers for longer duration of diagnosed HIV infection. Individuals in the POPPY study represent a selected group of PWH, many of whom have been diagnosed for substantial periods of time and have received multiple ART regimens, including some no longer in regular use. Thus, findings relating to ART exposure are likely to differ in this cohort to those from cohorts of newly diagnosed individuals starting cART. In Koeppe's study of PWH diagnosed with chronic pain, cART was associated with decreasing rates of pain over a median follow-up period of 5.2 years [13]. Among PWH attending a routine outpatient appointment in the UK in 2007 [14], the only HIV factors significantly associated with current pain in multivariable analyses were a longer time since HIV diagnosis and current receipt of a PI. Any PIs in use at the time of this study would have been earlier generation PIs with a different toxicity profile to those in common use today.

One finding from our study was the increased risk of pain in those with lower educational attainment. Although this is unlikely to reflect a causal association, instead reflecting unmeasured socio-economic status and lifestyle/behavioural factors, this finding does provide a means to identify a group at increased risk of experiencing pain and in need of specific support.

As yet, the mechanisms by which HIV may lead to an increased risk of pain remain unclear. Although biological pathways have been proposed [28], in 2013 Merlin widened understanding of pain cause and impact with a conceptual biopsychosocial framework of chronic pain in PWH [29]. This included biological (e.g. HIV neuropathy), psychological (following traumatic events) and social (environmental and relationship) factors. These primary studies and conceptual models highlight the need to identify and treat pain among PWH. We have recently used qualitative data from a study of PWH in the UK to adapt the Fear Avoidance pain model [30,31] to explain 
how stigma and attribution of pain to HIV lead to disengagement from both formal healthcare services and social support networks (paper in preparation).

Our analyses used an approach to define widespread pain based on both the number of sites affected and the variation of these across the body. There are many definitions of widespread pain based on the location, distribution, and number of painful body locations/sites affected [32]. The original criteria for widespread pain, developed in the context of fibromyalgia diagnosis, considered pain to be widespread if it was present in both the right and left sides of the body, both above and below the waist, and in the axial skeletal plane. However, this definition was criticised because individuals could meet this criterion whilst reporting pain in only a few sites, leading to additional proposed criteria [32-34]. Our definition follows the latest (2019) American College of Rheumatology/Fibromyalgia criteria [19]. Notably, chest, abdominal and head pain are not generally evaluated in assessments for chronic widespread pain, and thus were not considered in this analysis. Head pain, in particular, is relatively common in PWH (reported by just over 6\% of the PWH in the present study), although is more common in those with widespread pain and thus its inclusion would likely have only minimal impact on our classification of pain.

Our study benefits from a large sample of PWH which is broadly representative of older PWH in western European settings and in the context of high levels of viral suppression, allowing us to describe the distribution and extent of pain among PWH in an era of very effective treatment. In comparison to many earlier studies, our study also benefits from the inclusion of appropriately selected HIV-negative controls with similar characteristics to the older PWH in the study, allowing us to determine the role of HIV infection in the development of pain. However, whilst the POPPY study is a prospective cohort, the analyses reported herein relate to the baseline visit only limiting inferences around causality, and we cannot rule out the possibility that unmeasured causes of pain may exist, which may introduce confounding. Furthermore, our data collection does not permit an assessment of pain chronicity, intensity or severity by site. Our analyses do not consider the potential impact on quality-of-life of pain at any specific site (nor whether this differs between those with and without HIV infection). Our sample of PWH includes MSM and heterosexual men and women of white or black 
African ethnicity, and our findings therefore may have limited generalisability to those of other ethnic groups or to those who acquired HIV through non-sexual routes.

Furthermore, participation in the study may have resulted in the selection of a sample of individuals who had particular concerns around ageing and thus reported rates of pain may differ from those who were not included in the study. However, pain is only one of several aspects of ageing that is being studied, and study recruitment materials do not identify pain as a specific focus of interest - thus, we believe that any potential bias is likely to be small. Information on pain was collected over the period 2013-2016 - it is possible that with the use of newer, and less toxic ART drugs, pain patterns and the impact of these, may have changed. However, feedback from our community representatives suggests that pain remains a major, understudied cause of concern to many of those living with HIV, regardless of demographics or study participation.

In conclusion, widespread pain is highly prevalent in PWH and appears to be related to longer duration of HIV infection or ART exposure, including amongst those who are currently virally suppressed on ART. Pain has been identified as a priority outcome for PWH [35]. Given the increasing focus on the attainment of the highest possible qualityof-life in this population that is ageing with HIV [36], our findings highlight the need for clinicians to be alert to possible chronic and severe pain in $\mathrm{PWH}$, particularly those who may have been on long-term suppressive ART, and for the development of effective approaches to support the management of pain in this population.

\section{Acknowledgements}

We would like to thank all participants and staff involved in the POPPY study.

POPPY management team: Marta Boffito, Paddy Mallon, Frank Post, Caroline Sabin, Memory Sachikonye, Alan Winston, Amalia Ndoutoumou, Daphne Babalis.

POPPY Scientific Steering Committee: Jane Anderson, David Asboe, Marta Boffito, Lucy Garvey, Paddy Mallon, Frank Post, Anton Pozniak, Caroline Sabin, Memory Sachikonye, Jaime Vera, Ian Williams, Alan Winston. 
POPPY Sites and Trials Unit (alphabetical): Caldecot Centre, King's College Hospital (Frank Post, Lucy Campbell, Selin Yurdakul, Sara Okumu, Louise Pollard, Beatriz Santana Suarez), Department of Infection and Population Health, University College London (Ian Williams, Damilola Otiko, Laura Phillips, Rosanna Laverick, Michelle Beynon, Anna-Lena Salz, Abigail Severn), Elton John Centre, Brighton and Sussex University Hospital (Martin Fisher, Amanda Clarke, Jaime Vera, Andrew Bexley, Celia Richardson, Sarah Kirk, Rebecca Gleig), HIV Molecular Research Group, School of Medicine, University College Dublin (Paddy Mallon, Alan Macken, Bijan Ghavani-Kia, Joanne Maher, Maria Byrne, Ailbhe Flaherty, Aoife McDermott), Homerton Sexual Health Services, Homerton University Hospital (Jane Anderson, Sifiso Mguni, Rebecca Clark, Rhiannon Nevin-Dolan, Sambasivarao Pelluri), Ian Charleson Day Centre, Royal Free Hospital (Margaret Johnson, Nnenna Ngwu, Nargis Hemat, Anne Carroll, Sabine Kinloch, Mike Youle and Sara Madge), Imperial Clinical Trials Unit, Imperial College London (Amalia Ndoutoumou, Daphne Babalis), St. Mary's Hospital London, Imperial College Healthcare NHS Trust (Alan Winston, Lucy Garvey, Jonathan Underwood, Lavender Tembo, Matthew Stott, Linda McDonald, Felix Dransfield), St Stephen's Centre, Chelsea and Westminster Hospital (Marta Boffito, David Asboe, Anton Pozniak, Margherita Bracchi, Nicole Pagani, Maddalena Cerrone, Daniel Bradshaw, Francesca Ferretti, Chris Higgs, Elisha Seah, Stephen Fletcher, Michelle Anthonipillai, Ashley Moyes, Katie Deats, Irtiza Syed, Clive Matthews, Peter Fernando).

POPPY methodology/ statistics/analysis: Caroline Sabin, Davide De Francesco, Emmanouil Bagkeris.

Author contributions: CAS, AW, MB, FAP, MS and PWGM designed and obtained funding for the study and are members of the POPPY study management team. CAS performed all data analyses, undertook a literature search and prepared the first draft of the manuscript. DB provided study co-ordination and, together with EB and AG, supported essential data collection and preparation of the datasets. RH, KN, FAP and MB provided expert input to the interpretation of pain and clinical data. JA, IW, JV and MJ provided intellectual input to the POPPY study design, supported study recruitment, data collection and clinical management. MS provided liaison with the HIV patient 
community for all aspects of the study design and management. All authors provided critical review of the draft manuscript and have seen and approved the final version.

\section{References}

1. May MT, Gompels M, Delpech V, Porter K, Orkin C, Klegg S, et al. Impact on life expectancy of HIV-1 positive individuals of CD4+ cell count and viral load response to antiretroviral therapy. AIDS 2014; 28:1193-1202.

2. Marcus JL, Chao CR, Leyden WA, Xu L, Quesenberry CP, Klein DB, et al. Narrowing the gap in life expectancy between HIV-infected and HIVuninfected individuals with access to care. J Acquir Imm Defic Syndr 2016; 73:39-46.

3. Smit M, Brinkman K, Geerlings S, Smit C, Thyagarajan K, Sighem Av, et al. Future challenges for clinical care of an ageing population infected with HIV: a modelling study. Lancet Infect Dis 2015; 15:810-818.

4. da Silva JG, da Rocha Morgan DA, Melo FCM, Dos Santos IK, de Azevedo KPM, de Medeiros HJ, et al. Level of pain and quality of life of people living with HIV/AIDS pain and quality of life in HIV/AIDS. AIDS Care 2017; 29:10411048.

5. Scott W, Arkuter C, Kioskli K, Kemp H, McCracken LM, Rice ASC, et al. Psychosocial factors associated with persistent pain in people with HIV: a systematic review with meta-analysis. Pain 2018; 159:2461-2476.

6. Sabin CA, Harding R, Bagkeris E, Nkhoma K, Post FA, Sachikonye M, et al. Pain in people living with HIV and its association with healthcare resource use, well being and functional status. AIDS 2018; 32:2697-2706.

7. Moyle GJ, Sadler M. Peripheral neuropathy with nucleoside antiretrovirals: risk factors, incidence and management. Drug Saf 1998; 19:481-494. 
8. Margolis AM, Heverling $\mathrm{H}$, Pham PA, Stolbach A. A review of the toxicity of HIV medications. Med Toxicol 2014; 10:26-39.

9. Kroenke K, Spitzer RL, Williams JB. The PHQ-9: validity of a brief depression severity measure. J Gen Intern Med 2001; 16:606-613.

10. Koeppe J, Lyda K, Johnson S, Armon C. Variables associated with decreasing pain among persons living with human immunodeficiency virus. Clin J Pain 2012; 28:32-38.

11. Merlin JS, Cen L, Praestgaard A, Turner M, Obando A, Alpert C, et al. Pain and physical and psychological symptoms in ambulatory HIV patients in the current treatment era. J Pain Symptom Manage 2012; 43:638-645.

12. Uebelacker LA, Weisberg RB, Herman DS, Bailey GL, Pinkston-Camp MM, Stein MD. Chronic pain in HIV-infected patients: relationship to depression, substance use, and mental health and pain treatment. Pain Med 2015; 16:18701881.

13. Tsui JI, Cheng DM, Coleman SM, Blokhina E, Gnatienko N, Bryant K, et al. Pain and risk behaviors among HIV-Infected persons in St. Petersburg, Russia. AIDS Behav 2017; 21:1775-1781.

14. Lawson E, Sabin C, Perry N, Richardson D, Gilleece $\mathrm{Y}$, Churchill $\mathrm{D}$, et al. Is HIV painful? An epidemiological study of the prevalence and risk factors for pain in HIV-infected patients. Clin J Pain 2015; 31:813-819.

15. Richardson JL, Heikes B, Karim R, Weber K, Anastos K, Young M. Experience of pain among women with advanced HIV disease. AIDS Pat Care \& STDS 2009; 23:503-511.

16. Bagkeris E, Burgess L, Mallon PW, Post FA, Boffito M, Sachikonye M, et al. Cohort profile: the Pharmacokinetic and clinical Observations in PeoPle over fiftY (POPPY) Study. Int J Epidemiol 2018; 47:1391-1392e. 
17. UK CHIC Study Steering Committee. The creation of a large UK-based multicentre cohort of HIV-infected individuals: The UK Collaborative HIV Cohort (UK CHIC) Study. HIV Med 2004; 5:115-124.

18. McGettrick P, Ghavami-Kia B, Tinago W, Macken A, O'Halloran J, Lambert JS, et al. The HIV Care Cascade and sub-analysis of those linked to but not retained in care: the experience from a tertiary HIV referral service in Dublin Ireland. HIV Clin Trials 2017: 18:93-95.

19. Wolfe F, Butler SH, Fitzcharles MA, Hauser W, Katz RL, Mease PJ, et al.

Revised chronic widespread pain criteria: development from and integration with fibromyalgia criteria. Scand J Pain 2019; doi.org/10.1515/sjpain-20190054.

20. Parker R, Stein DJ, Jelsma J. Pain in people living with HIV/AIDS: a systematic review. J Int AIDS Soc 2014; 17:18719.

21. Jiao JM, Jebakumar J, George MC, Simpson DM, Robinson-Papp J. Chronic pain disorders in HIV primary care: clinical characteristics and association with healthcare utilization. Pain 2016; 157:931-937.

22. Miaskowski C, Penko JM, Guzman D, Mattson JE, Bangsberg DR, Kushel MB. Occurrence and characteristics of chronic pain in a community-based cohort of indigent adults living with HIV infection. J Pain 2011; 12: 1004-1016.

23. Tsui JI, Cheng DM, Quinn E, Bridden C, Merlin JS, Saitz R, et al. Pain and mortality risk in a cohort of HIV-infected persons with alcohol use disorders. AIDS Behav 2016; 20:583-589.

24. Aouizerat BE, Miaskowski CA, Gay C, Portillo CJ, Coggins T, Davis H, et al. Risk factors and symptoms associated with pain in HIV-infected adults. $J$ Assoc Nurses AIDS Care 2010; 21:125-133.

25. Martin C, Pehrsson P, Osterberg A, Sonnerborg A, Hansson P. Pain in ambulatory HIV-infected patients with and without intravenous drug use. Eur J Pain 1999; 3:157-164. 
26. Evans SR, Ellis RJ, Chen $\mathrm{H}$, et al. Peripheral neuropathy in HIV: prevalence and risk factors. AIDS 2011; 25:919-928.

27. Chen H, Clifford DB, Deng L, Yeh TH, Lee AJ, Schifitto G, et al. Peripheral neuropathy in ART-experienced patients: prevalence and risk factors. $J$ Neurovirol 2013; 19:557-564.

28. Merlin JS, Westfall AO, Heath SL, Goodin BR, Stewart JC, Sorge RE, et al. IL-1B levels are associated with chronic multisite pain in people living with HIV. $J$ Acquir Immune Defic Syndr 2017; 75:e99-e103.

29. Merlin JS, Zinski A, Norton WE, Ritchie CS, Saag MS, Mugavero MJ, et al. A conceptual framework for understanding chronic pain in patients with HIV. Pain Practice 2013; 14:207-216.

30. Crombez G, Eccleston C, Van Damme S, Vlaeyen JW, Karoly P. Fear-avoidance model of chronic pain: the next generation. Clin J Pain 2012; 28:475-483.

31. Vlaeyen JW, Linton SJ. Fear-avoidance and its consequences in chronic musculoskeletal pain: a state of the art. Pain 2000; 85:317-332.

32. Wolfe F, Butler SH, Fitzcharles MA, Hauser W, Katz RL, Mease PJ, et al. Revised chronic widespread pain criteria: development from and integration with fibromyalgia criteria. Scand J Pain 2019; doi.org/10.1515/sjpain-2019-0054.

33. Wolfe F, Clauw DJ, Fitzcharles M-A, Goldenberg DL, Hauser W, Katz RL, et al. 2016 Revisions to the 2010/2011 fibromyalgia diagnostic criteria. Semin Arthritis Rheum 2016; 46:319-329.

34. Wolfe F, Clauw D, Fitzcharles MA, Goldenberg DL, Katz RS, Mease P, et al. The American College of Rheumatology Preliminary Diagnostic Criteria for Fibromyalgia and measurement of symptom severity. Arthritis Care Res 2010; 62:600-610.

35. Wolfe F, Clauw D, Fitzcharles MA, Goldenberg DK, Hauser W, Katz RS, et al. Fibromyalgia criteria and severity scales for the clinical and epidemiological 
studies: a modification of the ACR preliminary diagnostic criteria for fibromyalgia. J Rheumatol 2011; 38:1113-1122.

36. Bristowe K, Clift P, James R, Josh J, Platt M, Whetham J, et al. Towards personcentred care for people living with HIV: what core outcomes matter, and how might we assess them? A cross-national multi-centre qualitative study with key stakeholders. HIV Med 2019; 20:542-554.

37. Safreed-Harmon K, Anderson J, Azzopardi-Muscat N, Behrens GWN, d'Arminio Monforte A, Davidovich U, et al. Reorienting health systems to care for people with HIV beyond viral suppression. Lancet HIV 2019; 6:e869-e877. 
Table 1: Characteristics of POPPY participants included in analyses of pain manikin data, overall and stratified by POPPY study group, and of the subgroup of PWH with complete ART data; table entries show $n$ and column percentages, unless otherwise stated

\begin{tabular}{|c|c|c|c|c|c|c|c|}
\hline & & \multicolumn{2}{|c|}{$\begin{array}{c}\text { Participants with } \\
\text { eligible pain manikin } \\
\text { data }\end{array}$} & \multicolumn{3}{|c|}{ POPPY study group } & \multirow[t]{2}{*}{$\begin{array}{l}\text { PWH with } \\
\text { complete } \\
\text { ART data }\end{array}$} \\
\hline & & Yes & No & $\begin{array}{l}\mathrm{PWH} \geq 50 \\
\text { years }\end{array}$ & $\begin{array}{l}\text { PWH }<50 \\
\text { years }\end{array}$ & $\begin{array}{l}\text { HIV- } \\
\text { negative } \\
\geq 50 \text { years }\end{array}$ & \\
\hline \multicolumn{2}{|c|}{ Number of participants } & $\begin{array}{l}1207 \\
(100.0)\end{array}$ & $\begin{array}{l}170 \\
(100.0)\end{array}$ & $\begin{array}{l}614 \\
(100.0)\end{array}$ & $\begin{array}{l}330 \\
(100.0)\end{array}$ & $\begin{array}{l}263 \\
(100.0)\end{array}$ & $\begin{array}{l}907 \\
(100.0)\end{array}$ \\
\hline \multirow[t]{2}{*}{ Gender } & Male & $990(82.0)$ & $\begin{array}{l}119 \\
(70.0)\end{array}$ & $547(89.1)$ & $269(81.5)$ & $174(86.2)$ & 785 (86.6) \\
\hline & Female & $217(18.0)$ & $51(30.0)$ & $67(10.9)$ & $61(18.5)$ & $89(33.8)$ & $122(13.5)$ \\
\hline \multirow{2}{*}{$\begin{array}{l}\text { Sexuality/risk } \\
\text { group }\end{array}$} & MSM & 865 (71.7) & $98(57.7)$ & $494(80.5)$ & $242(73.3)$ & $129(49.1)$ & $708(78.1)$ \\
\hline & Heterosexual & $342(28.3)$ & $72(42.4)$ & $120(19.5)$ & $88(26.7)$ & $134(51.0)$ & 199 (21.9) \\
\hline \multirow[t]{2}{*}{ Race } & White & $\begin{array}{l}1046 \\
(86.7)\end{array}$ & $\begin{array}{l}129 \\
(75.9)\end{array}$ & $542(88.3)$ & $268(81.2)$ & $236(89.7)$ & $777(85.7)$ \\
\hline & Black African & $161(13.3)$ & $41(24.1)$ & $72(11.7)$ & $62(18.8)$ & $27(10.3)$ & $130(14.3)$ \\
\hline \multirow{4}{*}{$\begin{array}{l}\text { Work } \\
\text { status }\end{array}$} & Employed & $648(53.7)$ & $64(37.7)$ & $251(40.9)$ & $232(70.3)$ & $165(62.7)$ & $459(50.6)$ \\
\hline & Student & $15(1$. & $1(0.6)$ & $4(0.7)$ & $10(3.0)$ & $1(0.4)$ & $14(1.5)$ \\
\hline & $\begin{array}{r}\text { Unemployed/off } \\
\text { sick }\end{array}$ & $263(21.8)$ & $22(12.9)$ & $170(27.7)$ & $75(22.7)$ & $18(6.8)$ & $237(26.1)$ \\
\hline & Other/unknown & $281(23.3)$ & $83(48.8)$ & $189(30.8)$ & $13(3.9)$ & $79(30.0)$ & $197(21.7)$ \\
\hline $\begin{array}{l}\text { Age } \\
\text { (years) }\end{array}$ & Median (IQR) & $\begin{array}{l}54(49, \\
60)\end{array}$ & $\begin{array}{l}54(49, \\
60)\end{array}$ & $56(53,62)$ & $\begin{array}{l}43(38, \\
47)\end{array}$ & $\begin{array}{l}58(53, \\
63)\end{array}$ & $\begin{array}{l}53(47, \\
59)\end{array}$ \\
\hline $\begin{array}{l}\text { BMI } \\
\left(\mathrm{kg} / \mathrm{m}^{2}\right)\end{array}$ & Median (IQR) & $\begin{array}{l}25.7 \\
(23.3 \\
28.7)\end{array}$ & $\begin{array}{l}25.7 \\
(23.3 \\
28.2)\end{array}$ & $\begin{array}{l}25.6 \\
(23.3 \\
28.5)\end{array}$ & $\begin{array}{l}25.2 \\
(23.0, \\
27.8)\end{array}$ & $\begin{array}{l}27.0 \\
(24.3 \\
29.7)\end{array}$ & $\begin{array}{l}25.6 \\
(23.2, \\
28.3)\end{array}$ \\
\hline \multirow{4}{*}{$\begin{array}{l}\text { Year of baselin } \\
\text { visit }\end{array}$} & 2013 & $174(14.4)$ & $20(11.8)$ & $106(17.3)$ & $57(17.3)$ & $11(4.2)$ & $159(17.5)$ \\
\hline & 2014 & $521(43.2)$ & $82(48.2)$ & $275(44.8)$ & $131(39.7)$ & $115(4$ & $391(43.1)$ \\
\hline & 2015 & $465(38.5)$ & $61(35.9)$ & $216(35.2)$ & $132(40.0)$ & $117(44.5)$ & $333(36.7)$ \\
\hline & 2016 & $47(3.9)$ & $7(4.1)$ & $17(2.8)$ & $10(3.0)$ & $20(7.6)$ & $24(2.7)$ \\
\hline \multirow{3}{*}{$\begin{array}{l}\text { Educational } \\
\text { attainment }\end{array}$} & Low & $317(26.3)$ & $26(15.3)$ & 179 (29.2) & $78(23.6)$ & $60(22.8)$ & $255(28.1)$ \\
\hline & Moderate & $170(14.1)$ & $23(13.5)$ & $82(13.4)$ & 55 (16.7) & 33 (12.6) & $129(14.2)$ \\
\hline & & $720(59.7)$ & $\begin{array}{l}121 \\
(71.2)\end{array}$ & $353(57.5)$ & 197 (59.7) & $170(64.6)$ & $523(57.7)$ \\
\hline
\end{tabular}


Table 2: Sites affected by pain, overall and stratified by POPPY study group

\begin{tabular}{|c|c|c|c|c|c|}
\hline \multirow[b]{3}{*}{ Body site } & \multirow{3}{*}{$\begin{array}{l}\text { Overall } \\
\mathrm{N}(\%)\end{array}$} & \multicolumn{3}{|c|}{ POPPY Study group } & \multirow{3}{*}{ P-value* } \\
\hline & & $\begin{array}{l}\mathrm{PWH} \geq 50 \\
\text { years }\end{array}$ & $\begin{array}{l}\mathrm{PWH}<50 \\
\text { years }\end{array}$ & $\begin{array}{l}\mathrm{HIV} \text {-ve } \geq 50 \\
\text { years }\end{array}$ & \\
\hline & & $\mathrm{N}(\%)$ & $\mathrm{N}(\%)$ & $\mathrm{N}(\%)$ & \\
\hline $\mathrm{N}$ & 1207 & 614 & 330 & 263 & \\
\hline Neck & $149(12.3)$ & $77(12.5)$ & $48(14.6)$ & $24(9.1)$ & 0.13 \\
\hline Upper back & $224(18.6)$ & $117(19.1)$ & $78(23.6)$ & $29(11.0)$ & 0.0004 \\
\hline Lower back & $359(29.7)$ & $195(31.8)$ & $92(27.9)$ & $72(27.4)$ & 0.29 \\
\hline Subtotal - region 1 & $426(35.3)$ & $228(37.1)$ & $116(35.2)$ & $82(31.2)$ & 0.24 \\
\hline Left shoulder & $193(16.0)$ & $108(17.6)$ & $49(14.9)$ & $36(13.7)$ & 0.28 \\
\hline Left upper arm & $101(8.4)$ & $57(9.3)$ & $25(7.6)$ & $19(7.2)$ & 0.50 \\
\hline Left lower arm & $157(13.0)$ & $94(15.3)$ & $36(10.9)$ & $27(10.3)$ & 0.05 \\
\hline Subtotal-region 2 & $299(24.8)$ & $172(28.0)$ & $72(21.8)$ & $55(20.9)$ & 0.03 \\
\hline Right shoulder & $203(16.8)$ & $119(19.4)$ & $46(13.9)$ & $38(14.5)$ & 0.05 \\
\hline Right upper arm & $105(8.7)$ & $60(9.8)$ & $29(8.8)$ & $16(6.1)$ & 0.21 \\
\hline Right lower arm & $164(13.6)$ & $100(16.3)$ & $41(12.4)$ & $23(8.8)$ & 0.009 \\
\hline Subtotal - region 3 & $317(26.3)$ & $187(30.5)$ & $76(23.0)$ & $54(20.5)$ & 0.003 \\
\hline Left hip & $94(7.8)$ & $57(9.3)$ & $23(7.0)$ & $14(5.3)$ & 0.11 \\
\hline Left upper leg & $349(28.9)$ & $191(31.1)$ & $88(26.7)$ & $70(26.6)$ & 0.23 \\
\hline Left lower leg & $310(25.7)$ & $181(29.5)$ & $72(21.8)$ & $57(21.7)$ & 0.009 \\
\hline Subtotal-region 4 & $500(41.4)$ & $282(45.9)$ & $124(37.6)$ & $94(35.7)$ & 0.005 \\
\hline Right hip & $119(9.9)$ & $76(12.4)$ & $27(8.2)$ & $16(6.1)$ & 0.008 \\
\hline Right upper leg & $338(28.0)$ & $182(29.6)$ & $85(25.8)$ & $71(27.0)$ & 0.41 \\
\hline Right lower leg & $296(24.5)$ & $178(29.0)$ & $66(20.0)$ & $52(19.8)$ & 0.001 \\
\hline Sub-total-region 5 & $493(40.9)$ & & $118(35.8)$ & $96(36.5)$ & 0.004 \\
\hline Total sites, median & 2 & 2 & 1 & 1 & \\
\hline IQR & $0-4$ & $0-5$ & $0-4$ & $0-3$ & \\
\hline Range & $0-15$ & $0-15$ & $0-15$ & $0-15$ & 0.002 \\
\hline Total regions, median & 1 & & 1 & 1 & \\
\hline IQR & $0-3$ & $0-3$ & $0-3$ & $0-2$ & \\
\hline Range & $0-5$ & $0-5$ & $0-5$ & $0-5$ & 0.0008 \\
\hline Pain extent, $\mathrm{n}(\%)$ & & & & & \\
\hline Widespread & $182(15.1)$ & $115(18.7)$ & $42(12.7)$ & $25(9.5)$ & \\
\hline Regional & $571(47.3)$ & $292(47.6)$ & $148(44.8)$ & $131(49.8)$ & \\
\hline Absent & $454(37.6)$ & $207(33.7)$ & $140(42.4)$ & $107(40.7)$ & 0.001 \\
\hline
\end{tabular}

* P-value obtained from Chi-squared test (Kruskal-Wallis test for total sites/regions) 
Table 3: Socio-demographic and lifestyle associations with widespread pain among PWH; table entries show $n$ and column percentages, unless otherwise stated

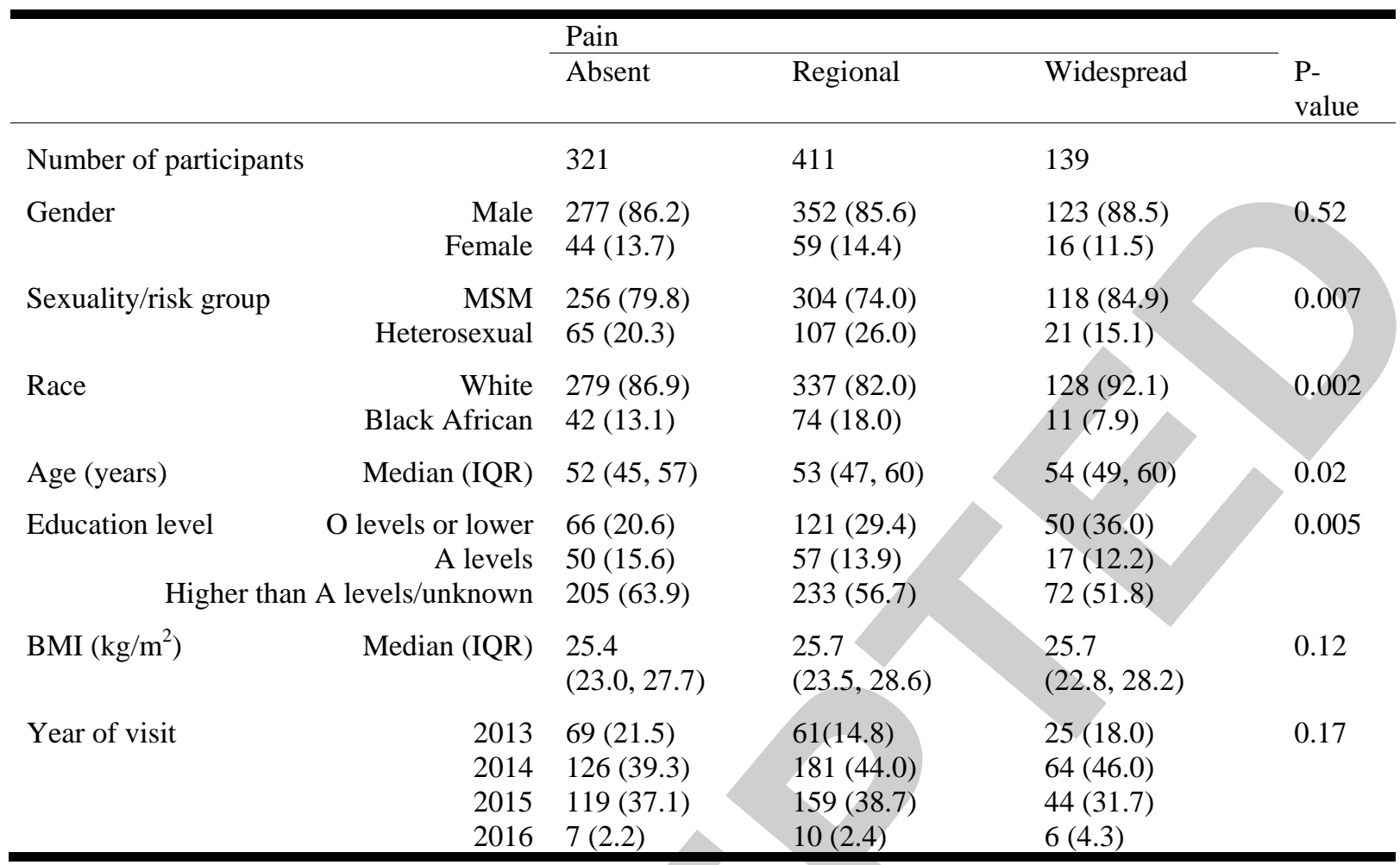

P-value obtained from Chi-squared test (categorical covariates) or Kruskal-Wallis test (continuous covariates)

Table 4: Associations between demographic, ART and immunosuppression factors and the extent of pain (widespread, regional or absent)

\begin{tabular}{lrll}
\hline & aOR (95\% CI) & p-value \\
\hline Educational attainment & O-levels or lower & $1.50(1.11-2.02)$ & \\
& A-levels & $0.95(0.66-1.39)$ & \\
& Higher/unknown & 1 & 0.02 \\
Total number of ART drugs received & (/drug) & $1.12(1.07-1.17)$ & 0.0001 \\
Current CD4 count & (/50 cells/mm $/ \mathrm{mm}^{3}$ higher) & $1.03(1.01-1.06)$ & 0.007 \\
Cumulative exposure to & (/year) & $1.07(1.01-1.14)$ & 0.03 \\
immunosuppression & & & \\
\hline
\end{tabular}

aOR: adjusted odds ratio; CI: confidence interval; ART: antiretroviral therapy 
Figure 1: Association between extent of pain and HIV-specific factors

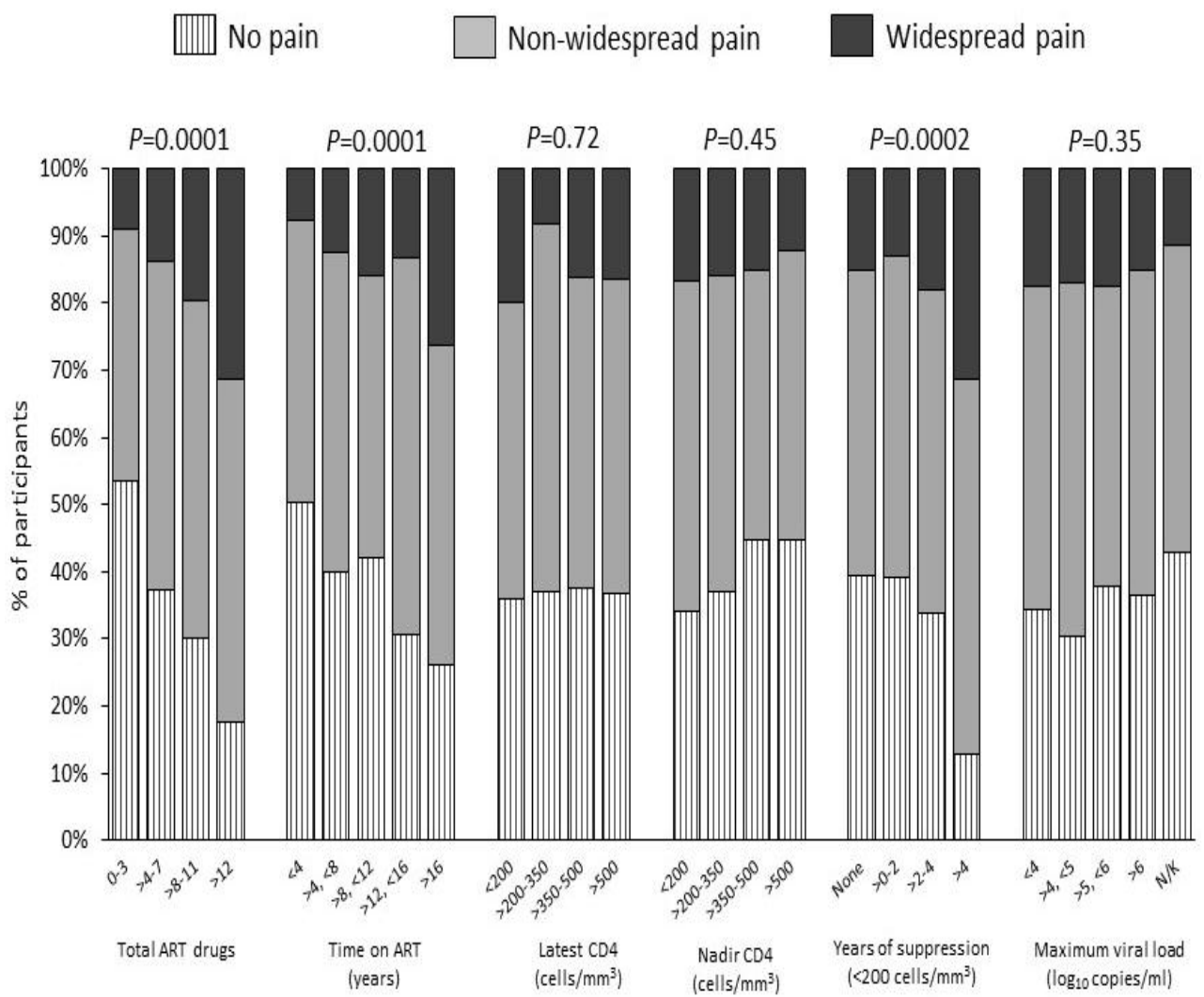

Copyright $\odot 2020$ Wolters Kluwer Health, Inc. Unauthorized reproduction of this article is prohibited. 